\title{
Performance evaluation of formulated de-emulsifier samples and a commercially available sample in the de-emulsification of a Nigerian crude oil emulsion
}

\author{
Efeovbokhan, Vincent Enon and Hymore, Fredrick Kofi \\ E-mail ghevej@gmail.com; phone number +2348052023977 \\ Akinola, Toluleke phone number +2348023004639 \\ E-Mail t.leke@yahoo.com \\ E-mail hymfred436@gmail.com; phone number +2347035009388 \\ Chemical Engineering Department, Covenant University, Ota, Nigeria.
}

\begin{abstract}
The de-emulsification of water-in-oil emulsion of Ogharefe crude oil sample was studied by using formulated polyester based de-emulsifier sample $\mathrm{A}$ and a commercially available de-emulsifier sample $C$. The bottle test method was used to screen the de-emulsifier samples using the crude oil emulsion. The performance of the de-emulsifiers was expressed in terms of percentage of water separated from $100 \mathrm{ml}$ samples of emulsions. For both the formulated and commercial deemulsifiers, the performance increased with increased concentration of the de-emulsifiers, separation time and operating temperature. The effect of the operating temperature was much higher and there was a linear relationship ( $R^{2}$ ranging from 0.96 to 0.99$)$ between performance and temperature. The performance of the best of the formulated de-emulsifiers, sample $A$, was better than that of the commercial de-emulsifier under all the conditions of this study- the volume of water expelled by sample $A$ was 5 times that of the commercial one at $30^{\circ} \mathrm{C}$. At $70^{\circ} \mathrm{C}$, this ratio increased to 14.
\end{abstract}

\section{INTRODUCTION}

Petroleum produced from the depths of the earth is associated with water or aqueous solutions of salts, particularly sodium chloride, in the form of emulsions. Emulsions are also produced during the processing of crudes and also during spills especially over waters. The presence of water in emulsions (in crudes) presents several problems during processing as well as during recovery-such as the increased viscosity and hence increased pumping costs, pipeline corrosions, hampering of skimmer operations, increased handling of oily waste disposal and storage problems etc. These emulsions may contain as much as $80 \%$ water and are frequently extremely stable due to the presence in the emulsion of a variety of emulsifiers such as asphaltenes. Asphaltenes are the heaviest and most polar fraction in the crude oil and play a leading role in causing the variety of nuisances such as the stabilization of the water-in-oil-emulsion that occurs during crude oil production (Auflem, 2002). They tend to adsorb at water-in-crude oil interfaces to form a rigid film surrounding the water droplets and protect the interfacial film from rupturing during droplet-droplet collisions, giving rise to the formation of particularly stable water-in-crude oil emulsion (Sjoblom, et al, 1990). Hence it is necessary to remove the water prior to processing and handling.

De-emulsification is the breaking or destabilization of crude oil emulsions in order to separate them into two clear immiscible phases i.e. oil and water phases. Of the four principal methods- mechanical, chemical, electrical and thermal-) (Selvarajan, et al, 2001) chemical de-emulsification is generally the most suitable method to break crude oil emulsions from both the operational and economic point of view. This is done by treating the emulsions with de-emulsifiers under different conditions of temperature and concentrations. The choice of de-emulsifiers to use for particular oil is usually from a selection of what have been used for other crudes. De-emulsifiers proposed for use may include organic substances such as sulphonates, polyglycol ethers, oxylated phenols, e.g. alkanolamine and nonylphenol ethoxylate derivatives (Easton, et al 1989) relatively recent additions include the organosiloxanes which are regarded as the most efficient for crude oil deemulsification. De-emulsifiers are interfacial-active agents which weaken the stabilizing films to enhance droplets coalescence. They are preferred to other 
chemical agents due to lower additions rates needed. The separation rate of W/O emulsion is usually a function of the de-emulsifier used, the emulsion stability, the temperature, the concentration, the process residence time and the mixing energy. (Sunil, 2006)

Because crudes vary in composition according to their location, the best de-emulsifier for a crude may not be same for another. Another factor affecting the performance of de-emulsifier is the water content of the emulsion. Research into the performance of deemulsifiers is thus a continuous process. In Nigeria where the industry is totally dependent on imported inputs for the oil and gas industry this search is even more important. In this study, polyester based deemulsifiers (Stephen, et al 1980) were tested for their performance in breaking emulsions of Nigerian crude under different conditions of temperature, concentration and separation time. Their comparison with that of commercial one will give a good measure of the performance of the formulated de-emulsifiers. A success will also serve to enhance the range of materials that can be used as de-emulsifiers of crude oils.

\section{MATERIALS AND METHODS}

\section{MATERIALS}

Crude oil emulsion sample for this study was obtained from an oil company situated in Delta State, Nigeria. A commercial de-emulsifier, sample C, (used as standard for comparison), was also obtained from the same company Polypropylene glycol, maleic anhydride and phosphoric acid (all of analar grade)

\section{EXPERIMENTAL METHODS}

The $250 \mathrm{ml}$ 3-neck round bottom flask to which was fixed a motorized mechanical stirrer, a thermometer, and a condenser for the removal of any aqueous phase or organic distillate formed in the course of the reaction, was placed in the $250 \mathrm{ml}$ sized heating mantle. Accurately weighed quantities of polypropylene glycol, maleic anhydride and phosphoric acid were then charged into the reactor. The set up was then heated at low stirring speed to the reaction temperature of $100^{\circ} \mathrm{C}$ and a residence time of four hours. The reaction mixture was then cooled and stored in an appropriately labelled sample bottle. The various weight ratios of the reacting materials resulted in the different products used for testing.

\section{DE-EMULSIFIER SCREENING}

The de-emulsifiers were screened using the bottle test method. The bottle test is an empirical test in which varying amounts of potential de-emulsifiers are added into a series of tubes or bottles containing the sample of an emulsion to be broken.

Here, eight centrifuge bottles were used for the screening test or experiment. $10 \mathrm{ml}$ of the emulsion sample was measured into the centrifuge bottles and a carefully determined quantity (in ppm) of the deemulsifier to be screened was added to the sample. Each sample was then stirred vigorously for 1 minute for proper mixing. The bottles were then contacted for 15 minutes in a water bath whose temperature had been preset to the test temperature. The bottles were then centrifuged at 1200 revolution per minute, rpm for different residence times for phase separation. The volume of water expelled from the emulsion system as a function of separation time and temperature were measured. The three de-emulsifier samples (A and B are formulated samples and sample $C$ is commercial), were all screened subject to the above procedures

\section{RESULTS AND DISCUSSION}

After the preliminary tests the performance of the two most promising formulated de-emulsifiers (samples $A$ and $B$ ) were compared with that of the commercial de-emulsifier (sample C) under different conditions of de-emulsifier concentration, separation time and temperature. The volume of water separated from the emulsion system as a function of operating conditions was used as a measure of the performance of a given de-emulsifier. This was expressed as:

\section{Por formance $-100 \times \frac{\text { volume of froter sepeated from the emulsion gytem }}{\text { volume of he emullion wystam }}$ 1) \\ EFFECT OF CONCENTRATION OF DE- EMULSIFIERS ON PERFORMANCE}

Three different concentrations of de-emulsifiers were used to examine the effects of the de-emulsifiers concentrations on rate of separation of the Ogharefe crude oil emulsion system. The concentrations used were 10ppm, 20ppm and 40ppm. Fig.1a, b, c, d and e illustrate the effects of concentrations of the formulated de-emulsifier sample $A$ and the separation 
Am. J. Sci. Ind. Res., 2010, 1(3): 629-635

time on its performance at different operating temperatures-30, 40,50, 60 and $70{ }^{\circ} \mathrm{C}$. The Figs. show a clear trend of these factors on the performance of the de-emulsifier sample $A$. The performance increased with increased separation time because more time had been allowed for the phases to separate. The separation time is crucial in the sizing of the separators for such processes, hence the importance in establishing its effect. At all temperatures, increasing the concentration of the deemulsifiers used had a significant effect as the performance improved with increased concentration. The effect of the concentration is indicative of the efficiency of the de-emulsifiers. The smaller the concentration required for a given effect, the more efficient the de-emulsifier and the better the economics of the separation process.

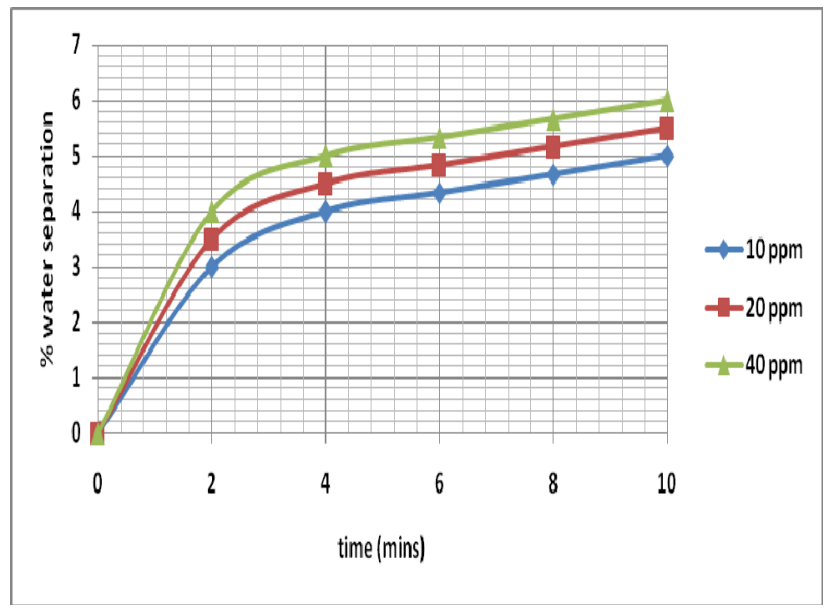

Fig.1a: The effect of concentrations of de-emulsifier ' $A$ ' and separation time on emulsion treatment at $30^{\circ} \mathrm{C}$

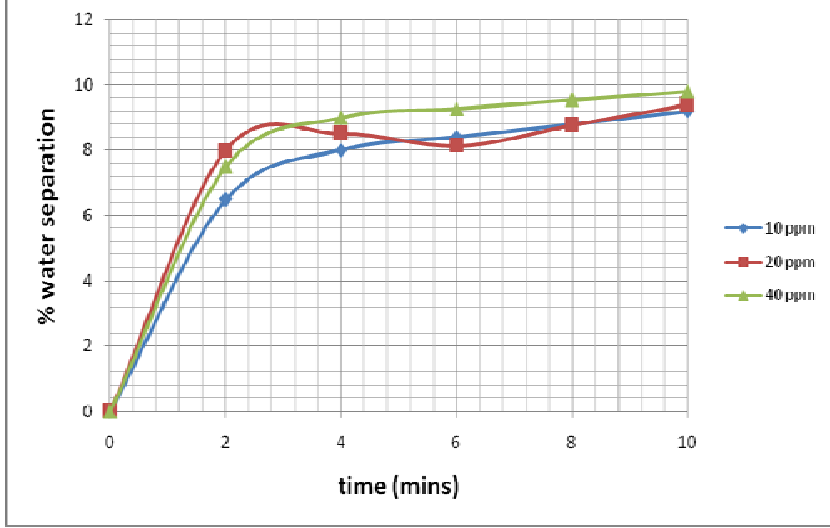

Fig.1b: The effect of concentrations of de-emulsifier ' $A$ ' and separation time on emulsion treatment at $40^{\circ} \mathrm{C}$

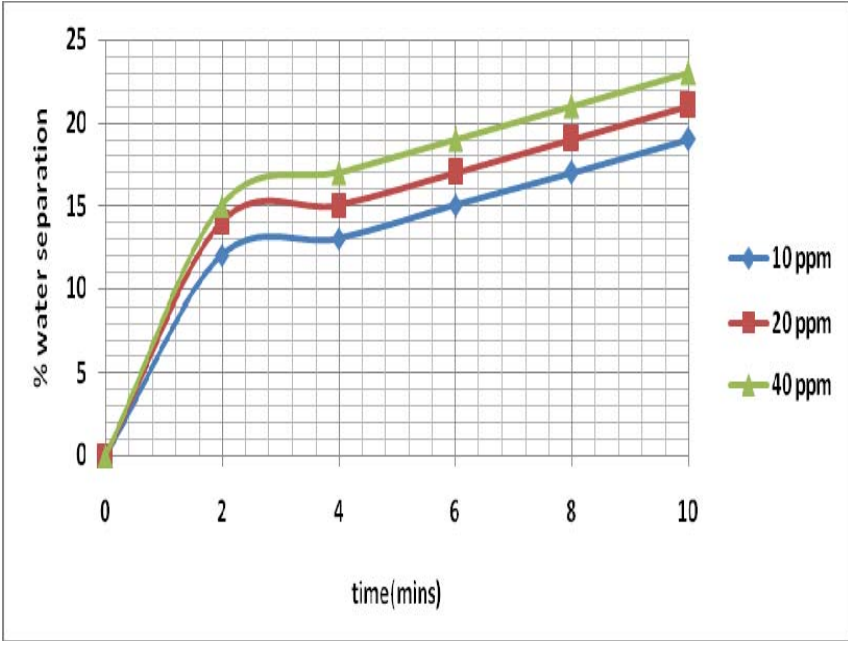

Fig.1c: The effect of concentrations of de-emulsifier ' $A$ ' and separation time on emulsion treatment at $50^{\circ} \mathrm{C}$

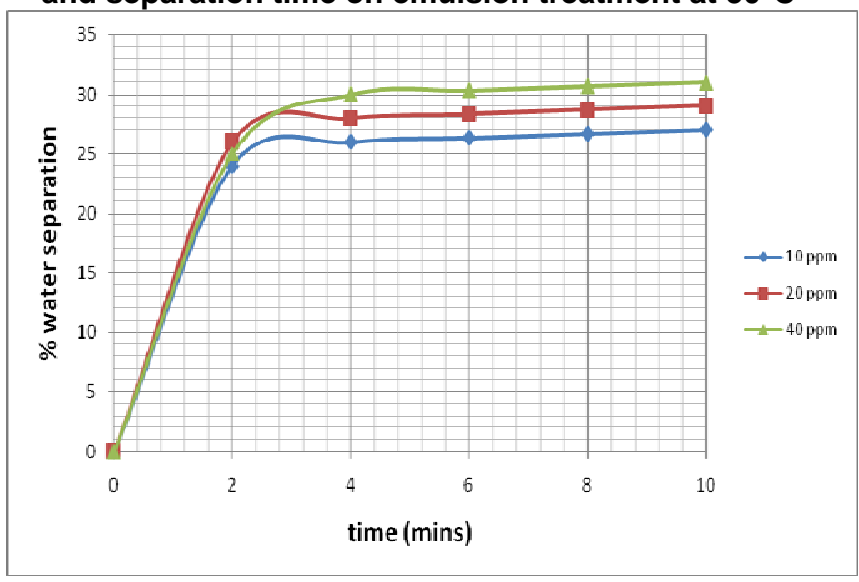

Fig.1d: The effect of concentrations of de-emulsifier ' $A$ ' and separation time on emulsion treatment at $60^{\circ} \mathrm{C}$

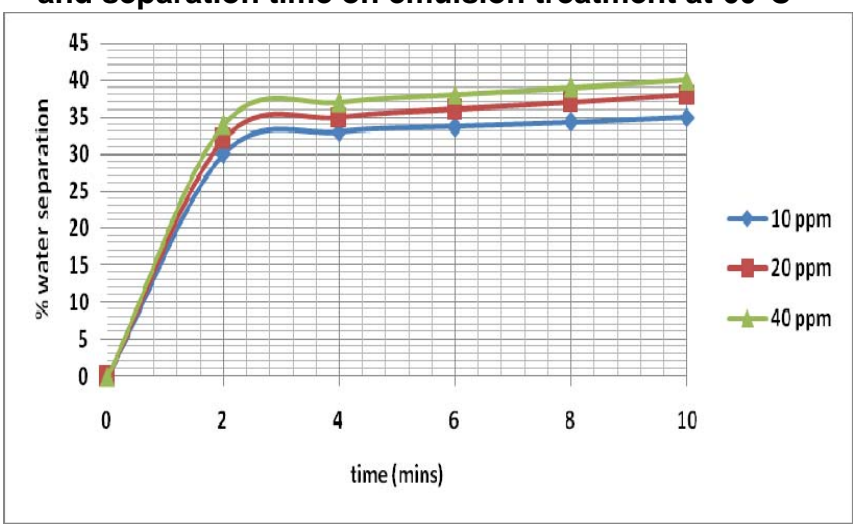

Fig.1e: The effect of concentrations of de-emulsifier ' $A$ ' and separation time on emulsion treatment at $70^{\circ} \mathrm{C}$ 
Am. J. Sci. Ind. Res., 2010, 1(3): 629-635

Fig.2a, b, c, d and e show similar performance trends for de-emulsifier ' $C$ 'as for de-emulsifier ' $A$ '. The performance increased with increased concentration and separation time at operating temperatures-30, $40,50,60$ and $70^{\circ} \mathrm{C}$. The extent of improvement is however not the same for each de-emulsifier.

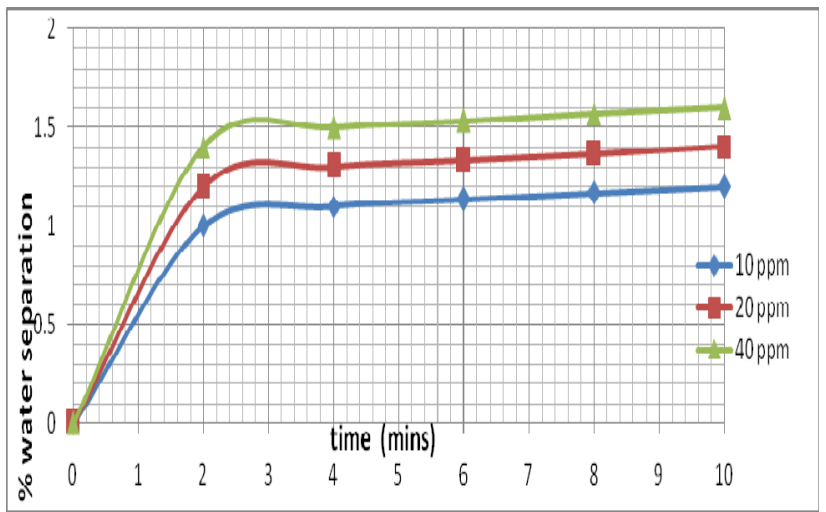

Fig.2a The effect of concentrations of de-emulsifier ' $C$ ' and separation time on emulsion treatment at $30^{\circ} \mathrm{C}$

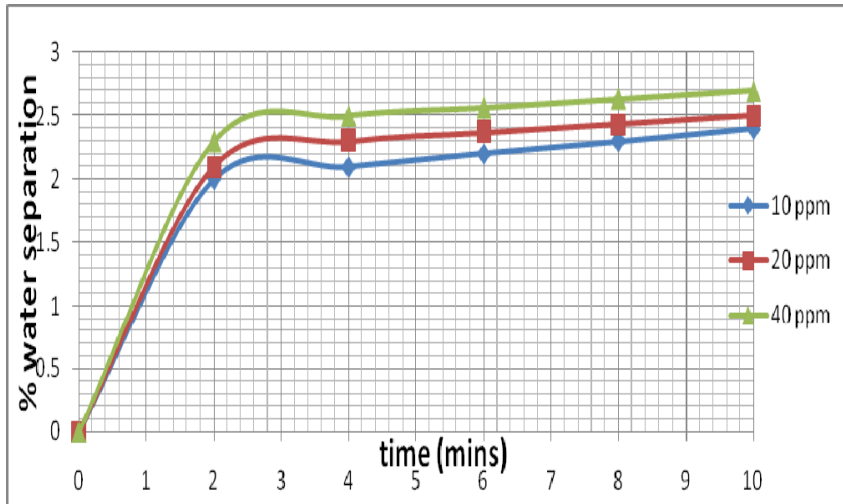

Fig.2b The effect of concentrations of de-emulsifier ' $C$ ' and separation time on emulsion treatment at $40^{\circ} \mathrm{C}$

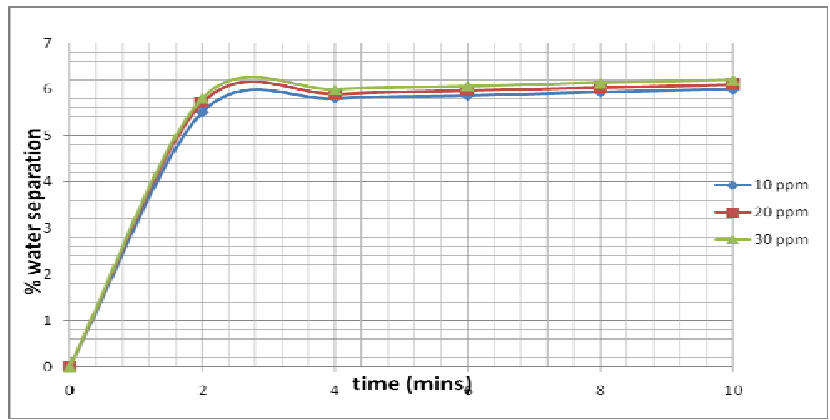

Fig.2c The effect of concentrations of de-emulsifier ' $C$ ' and separation time on emulsion treatment at $50^{\circ} \mathrm{C}$

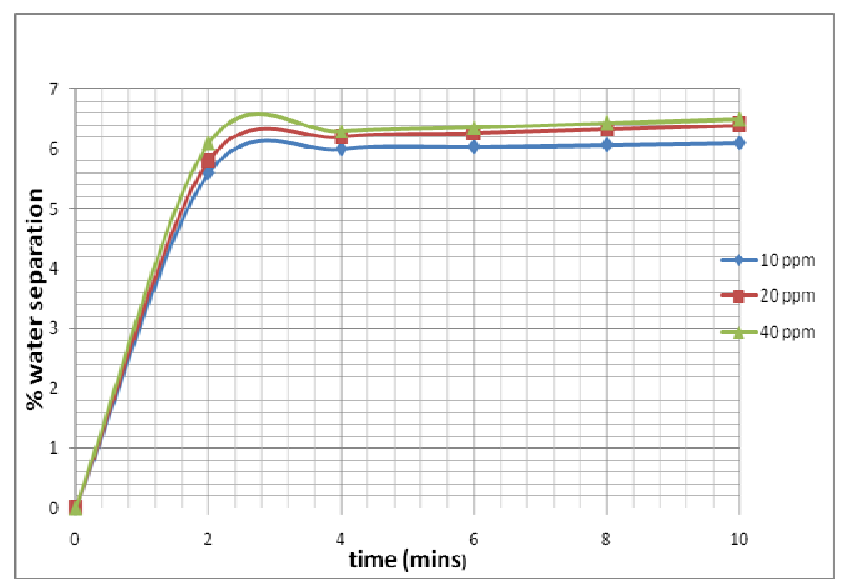

Fig. 2d: The effect of concentrations of de-emulsifier ' $C$ ' and separation time on emulsion treatment at $60^{\circ} \mathrm{C}$

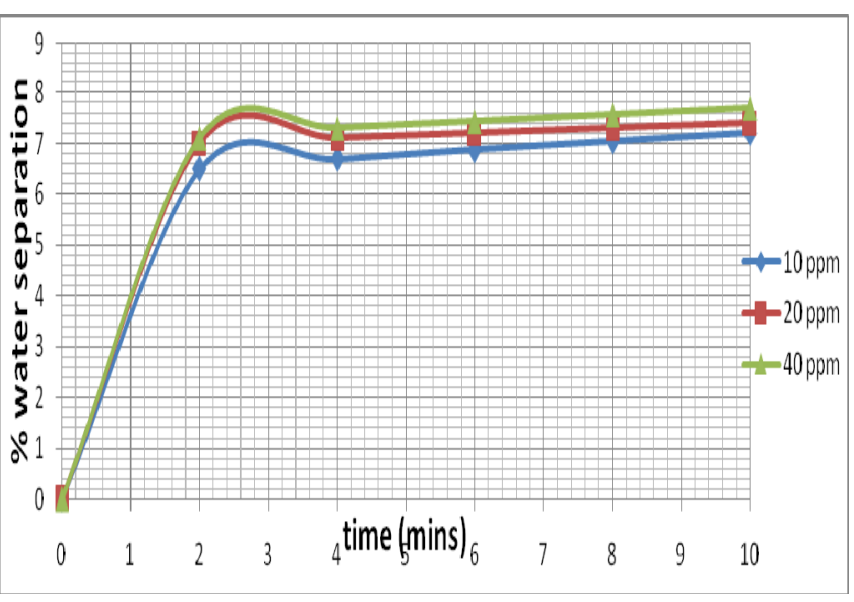

Fig. 2e: The effect of concentrations of de-emulsifier ' $C$ ' and separation time on emulsion treatment at $70^{\circ} \mathrm{C}$

\section{EFFECT OF TEMPERATURE ON DE-EMULSIFIER PERFORMANCE}

Fig. $3 a, 3 b$ and $3 c$ illustrate the effect of temperature on the performance of de-emulsifier ' $A$ ' at the three concentrations studied. All three Figs show that for a given separation time and concentrations of deemulsifier, there was a near-linear relationship between performance of de-emulsifier and the temperature of separation. The coefficient of correlation of the straight lines ranged from 0.96 to 0.99 . The yield of water from the separation process increased in all cases as the temperature was increased from $30^{\circ} \mathrm{C}$ to $70^{\circ} \mathrm{C}$. The percentage 
increase in water separated for a separation time of 10 minutes ranged from to 483 to 590 as the temperature was changed from $30^{\circ} \mathrm{C}$ to $70^{\circ} \mathrm{C}$. For a separation time of 4 minutes the percentage increase in water separated ranged from 640 to 725 for the same temperature change. These increases are indicative of the important influence of temperature on the separation process. At higher temperatures, the viscosities of the liquids decrease whiles their diffusivities increase. These two factors facilitate the movement of water out of the emulsions and thus enhance the performance of the de-emulsifiers.
The plots also show that the influence of the separation temperature is much more important than the separation time within the range used in this study. The effect of the separation time is seen readily from the increased slope of the straight lines as the separation time was increased from 4 to 10 minutes. The much smaller increase (compared to that of temperature) is due to the effect of the increased concentration of the de-emulsifier. This is seen from Table 1 which relates the slopes as a function of the concentrations of the de-emulsifiers.

Table 1: effect of de-emulsifier concentration and separation time on the performance

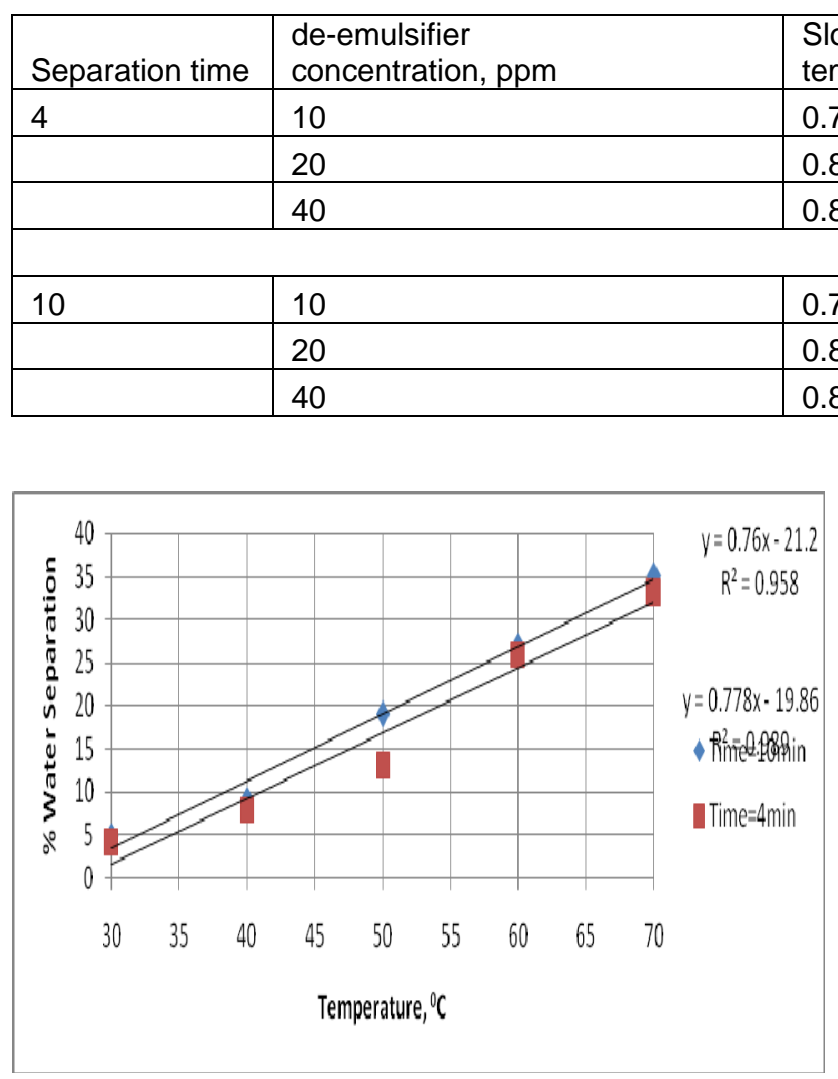

Fig.3a: The effect of separation temperature on the performance of de-emulsifier ' $A$ ' (10ppm)

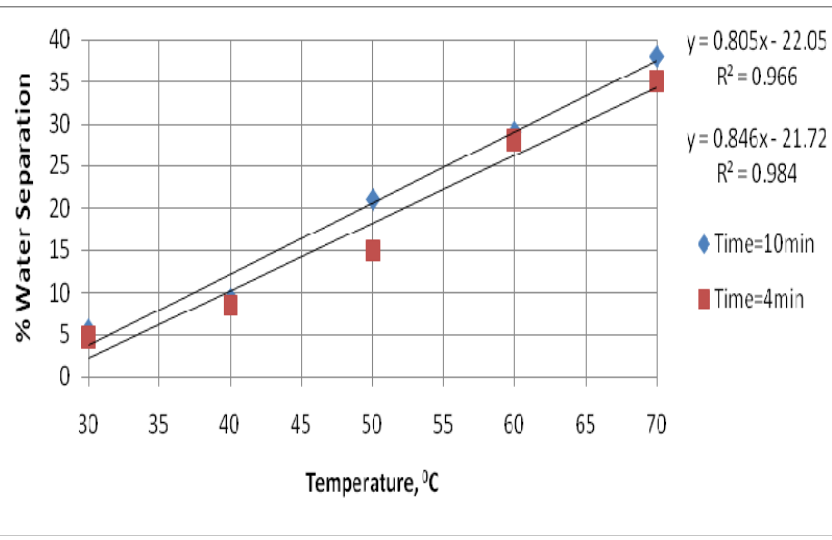

Fig.3b: The effect of separation temperature on the performance of de-emulsifier ' $A$ ' (20ppm) 


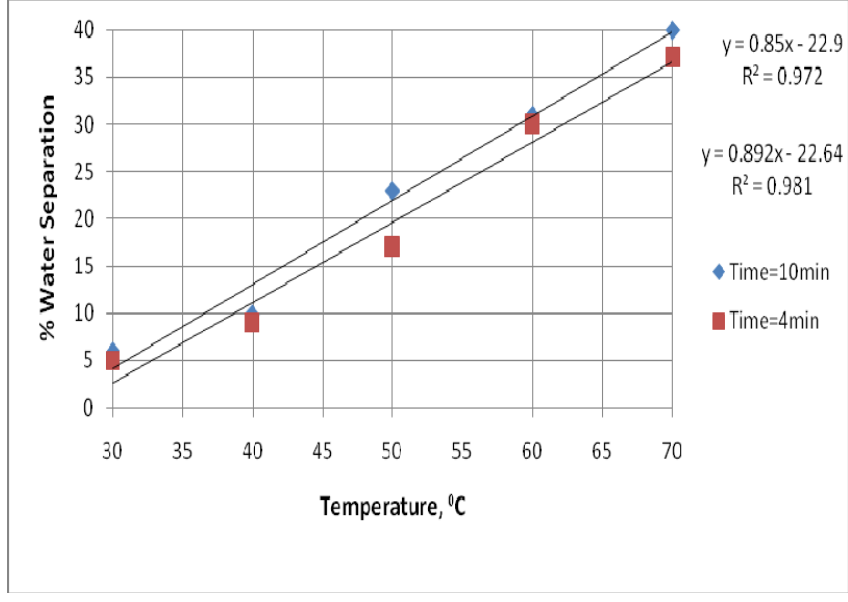

Fig.3c: The effect of separation temperature on the performance of de-emulsifier ' $A$ ' (40ppm)

\section{Comparison of the performances of formulated and a commercial de-emulsifiers}

The performances of the commercial and the formulated de-emulsifiers are compared under similar conditions as shown in Fig. $4 \mathrm{a}$ (at $30^{\circ} \mathrm{C}$ ) and $4 \mathrm{~b}$ $\left(70^{\circ} \mathrm{C}\right)$. Samples $A$ and $B$ were the best of the formulated de-emulsifiers whiles sample $C$ is the deemulsifier used by the company from which the oil sample for this study was obtained. Both the formulated and commercial all samples follow similar trends. The performances increased with increased separation time and higher separation temperature.

The extent of increase in performance however depended on the de-emulsifiers. At a de-emulsifier concentration of $10 \mathrm{ppm}$ and at a temperature of $30^{\circ} \mathrm{C}$ the performance of the commercial de-emulsifier was only slightly better than one of the formulated deemulsifiers (B) for separation times less than 10 minutes, at which point there was equality in performance. The best of the formulated deemulsifiers A) was much better than that of the commercial de-emulsifier within the separation times studied. At 10 minutes, the performance of the formulated de-emulsifiers $(A)$ was 5 times that of the commercial one-see Fig 4a.

At $70^{\circ} \mathrm{C}$, there was little difference between the performances of the commercial and sample B. There was however a marked improvement in performance of sample $A$ due to the temperature change $\left(30\right.$ to $70^{\circ} \mathrm{C}$ ). There was a seven fold increase in performance of sample A (5 to 35\%). At $70^{\circ} \mathrm{C}$, this represented nearly 14 times $(2.42 \%$ to $34 \%)$ compared to those of samples B and the commercial one-see Fig.4b. The same trend is illustrated in Fig.4.c where the improved performances of both the commercial and the best formulated may be attributed to the increased concentration of the de-emulsifiers. Even in this case the performance of the best of the formulated is still much better than the commercial one. It is thus clear that the best of the formulated de-emulsifiers A, gave a better performance than that of the commercial one under the experimental conditions used in the study.

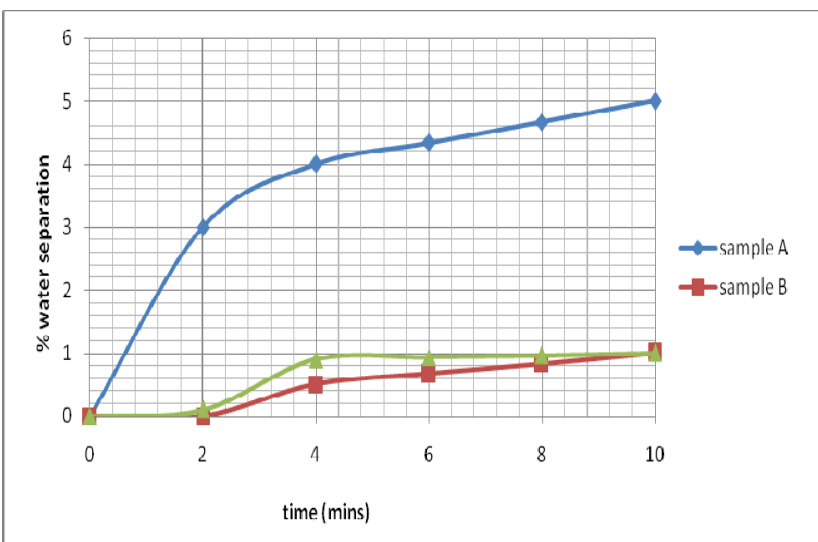

Fig 4a: Comparison of the performances of deemulsifiers at a concentration of $10 \mathrm{ppm}$ and at a temperature of $30^{\circ} \mathrm{C}$.

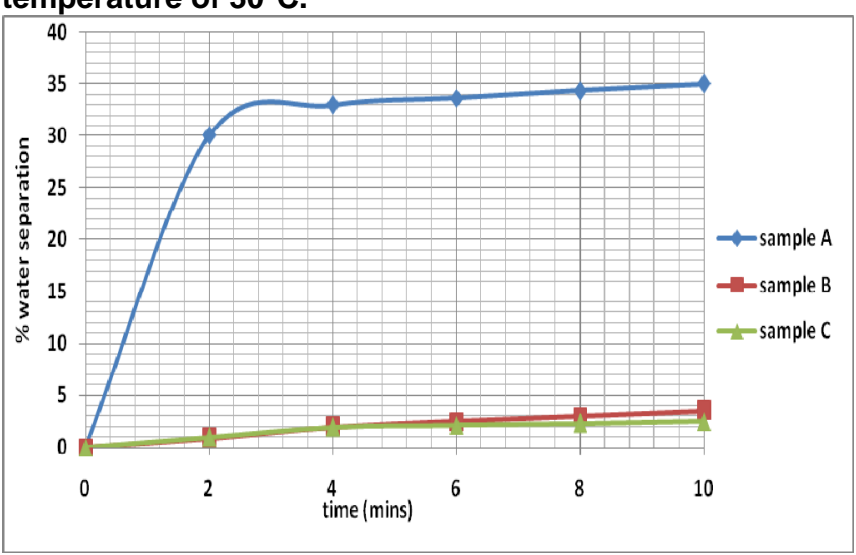

Fig 4b: Comparison of the performances of deemulsifiers at a concentration of $10 \mathrm{ppm}$ and at a temperature of $70^{\circ} \mathrm{C}$. 
Am. J. Sci. Ind. Res., 2010, 1(3): 629-635

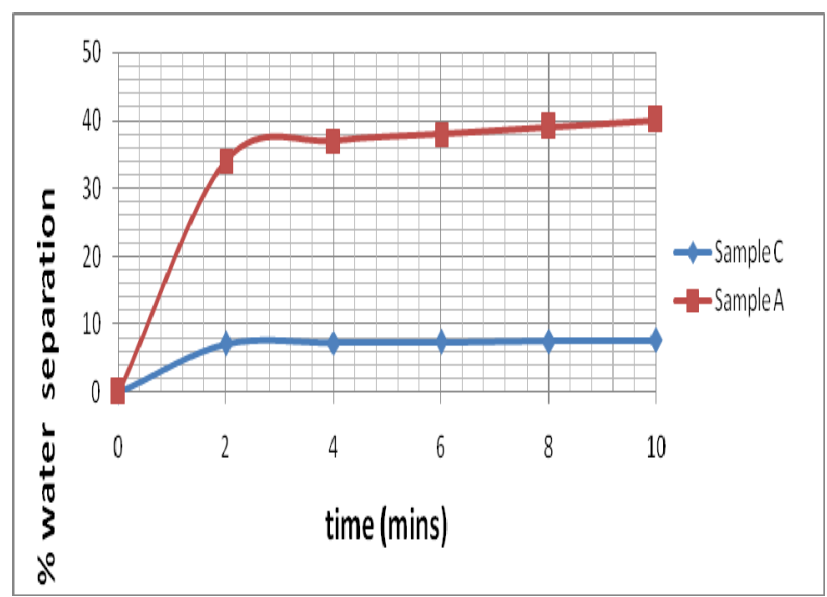

Fig 4c: Comparison of the performances of best formulated de-emulsifier and a commercial one at concentration of $40 \mathrm{ppm}$ and at a temperature of $70^{\circ} \mathrm{C}$.

\section{CONCLUSION:}

The study of performances of two formulated deemulsifiers (samples A and B) and a commercial sample (C) on a crude oil emulsion using the bottle test method revealed the following:

* At a constant temperature and separation time the performances of all de-emulsifiers increase with increase concentrations of the de-emulsifiers.

* Performance also generally increased with separation time at all concentrations and temperatures considered.

* The effect of temperature on performance, within all the ranges of temperatures studied, was greater than the corresponding effect of concentrations.

Performance increased linearly with operating temperature of de-emulsification $\left(R^{2}\right.$ ranging from 0.96 to 0.99$)$

* The effect of temperature on the performance of the best of the formulated deemulsifiers (sample A) was much higher than that of the commercial one (sample C)

\section{REFERENCES}

Aske, N., Kallevik, H. and Sjöblom, J. (2001). Determination of Saturate, Aromatic, Resin and
Asphaltenic (SARA) Components in Crude Oils by Means of Infrared and Near-Infrared Spectroscopy. Energy \& Fuels. 1304-1312.

Auflem, I. H. (2002). Influence of Asphaltene Aggregation and Pressure on Crude Oil Emulsion Stability. Norwegian University of Science and Technology. Doctor Ingeniør Thesis.

Easton Thomas, Thomas Bryan (1989), De- emulsification of oils, Free Patents Online All. The Inventions of Mankind http://www.freepatentsonline.com/4888107.html (viewed January, 2010)

Schorling, P. C., Kessel, D.G. and Rahimian, I. (1998). Influence of Crude Oil Resin/Asphaltene Ratio On The Stability of Oil/Water Emulsion. Colloids and Surfaces. German Petroleum Institute, German

Selvarajan Radhakrisnan, Ananthasubramaniam Sivakumar and Robert A. M. (2001). Aqueous Dispersion of An Oil Soluble Demulsifier For Breaking Crude Oil Emulsions. (US Patent No. 6,294,093).

Sjoblom, J., Ming Yuan, L., Hoiland, $\mathrm{H}$ and Johansen, J.E. (1990). Water-in-Crude Oil Emulsions from the Norwegian Continental Shelf, Part III. A Comparative Destabilization of Model Systems. Colloid and Surfaces. 46: 127-139.

Speight, J. G. (1991). The Chemistry and Technology of Petroleum. Marcel Dekker Inc. New York.

Stephen J. Rehm, Tulsa young R (1980). De-emulsifiers Free Patents Online All The Inventions Of Mankind http://www.freepatentsonline.com/4324734.pdf (viewed January, 2010)

Sunil L. Kokal, Saudi Aramco (2006) (SPE document) Crude Oil Emulsions, Petroleum Engineering Handbook Volume 1

http://balikpapan.spe.org/images/balikpapan/articles/51/Cru de\%200il\%20Emulsions\%20-\%20PEH $\% 20$ Chapter\%2012\%20-\%20compressed\%20version.pdf (viewed January, 2010).

Zaki, N.; Schorling, P.-C.; Rahimian, I. (2000). Petroleum Sci. Tech. 18: 945-963 